\title{
Statistical mechanical model of gas adsorption in a metal-organic framework harboring a rotaxane molecular shuttle
}

\author{
Jonathan Carney, ${ }^{\dagger}$ David Roundy, ${ }^{*, \dagger}$ and Cory M. Simon ${ }^{*, \dagger}$ \\ †Oregon State University. Department of Physics. Corvallis, OR, USA \\ $\ddagger$ Oregon State University. School of Chemical, Biological, and Environmental Engineering. \\ Corvallis, OR, USA \\ E-mail: roundyd@physics.oregonstate.edu; Cory.Simon@oregonstate.edu
}

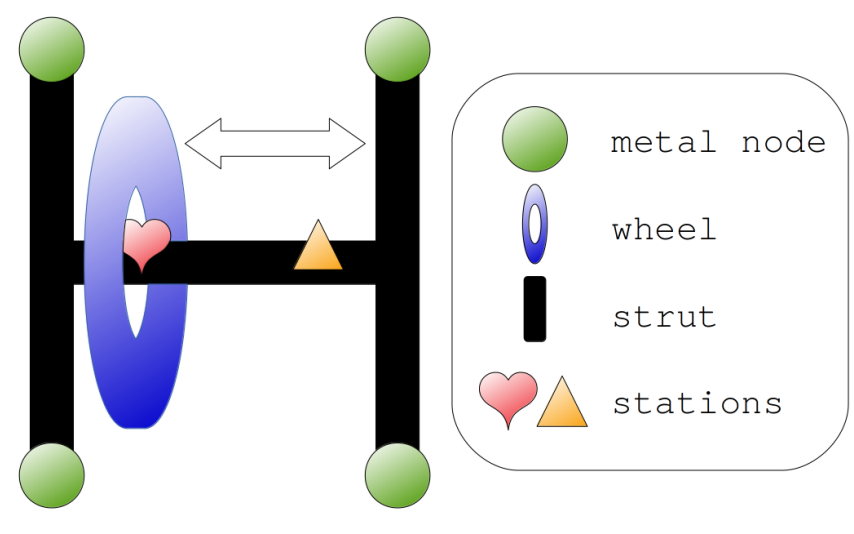

\section{Abstract}

Metal-organic frameworks (MOFs) are modular and tunable nano-porous materials with applications in gas storage, separations, and sensing. Flexible/dynamic components that respond to adsorbed gas can give MOFs unique or enhanced adsorption properties. Here, we explore the adsorption properties that could be imparted to a MOF by a rotaxane molecular shuttle (RMS) in its pores. In the unit cell of an RMS-MOF, a macrocyclic wheel is mechanically interlocked with a strut of the MOF scaffold. The wheel shuttles between stations on the strut that are also gas adsorption sites. At a level of abstraction similar to the seminal Langmuir adsorption model, we pose and analyze a simple statistical mechanical model of gas ad- sorption in an RMS-MOF that accounts for (i) wheel/gas competition for sites on the strut and (ii) gas-induced changes in the configurational entropy of the shuttling wheel. We determine how the amount of gas adsorbed, position of the wheel, and differential energy of adsorption depend on temperature, pressure, and the interactions of the gas/wheel with the stations. Our model reveals that, compared to a rigid, Langmuir material, the chemistry of the RMS-MOF can be tuned to render gas adsorption more or less temperature-sensitive and to release more or less heat upon adsorption. The model also uncovers a non-monotonic relationship between the temperature and the position of the wheel if gas out-competes the wheel for its preferable station.

\section{Introduction}

Metal-organic frameworks (MOFs) are nanoporous, crystalline materials with adsorption-based applications in storing, ${ }^{1}$ separating, ${ }^{2}$ and sensing ${ }^{3}$ gases. MOFs are synthesized modularly by linking metals or metal clusters, serving as nodes, to organic linker molecules, serving as struts. ${ }^{4,5}$ Because of the abundance of compatible molecular building blocks (nodes and struts), a practically limitless number of different MOFs, with diverse properties, can be synthesized (tens of thou- 
sands thus far ${ }^{6}$ ).

The integration of dynamic and flexible components into the structures of MOFs, enabled by their synthetic adjustability, could impart them with unique or enhanced adsorption properties that are inaccessible to static and rigid materials. ${ }^{7}$ Already, several different modes of flexibility have been incorporated in $\mathrm{MOFs}^{8-13}$ and given rise to anomalous adsorption properties ${ }^{14}$ that can be exploited for engineering applications. Modes of flexibility include the rotation ${ }^{15}$ and bending $^{16}$ of linkers, hinging of the backbone of e.g. wine-rack motifs, ${ }^{17}$ and displacement of interdigitated or interpenetrated networks; ${ }^{18}$ consequent anomalous adsorption properties ${ }^{14}$ include inflections ${ }^{19}$ and discontinuities ${ }^{9}$ in gas adsorption isotherms, ${ }^{13}$ intrinsic mitigation of the heat released upon adsorption, ${ }^{20}$ negative gas adsorption, ${ }^{21}$ adsorption-induced contraction $^{22}$ and expansion, ${ }^{20}$ metastability, ${ }^{23,24}$ and adsorption/desorption hysteresis. ${ }^{25}$

The MOF $\mathrm{Co}(\mathrm{bdp})^{25}$ is a flagship example of a flexible MOF with unique and useful adsorption properties. Co(bdp) possesses a winerack-like structure permissive of hinge motion. When immersed in methane gas, at low pressure, $\mathrm{Co}(\mathrm{bdp})$ adopts a collapsed, nonporous state, but expands to a porous state and fills with gas at higher pressures. ${ }^{20}$ This imparts Co(bdp) with several unique adsorption properties advantageous for vehicular natural gas storage and delivery. ${ }^{20}$ First, the resulting stepped methane adsorption isotherm equips $\mathrm{Co}(\mathrm{bdp})$ with the highest reported methane usable capacity. Unlike rigid MOFs, $\mathrm{Co}(\mathrm{bdp})$ collapses at the discharge pressure to fully expel its cushion gas. Second, the endothermic framework expansion induced by adsorbed gas consumes a fraction of the heat released upon adsorption. This is advantageous because such heat diminishes the usable capacity by raising the temperature of the adsorbent. ${ }^{26}$ Third, Co(bdp) responds to mechanical pressure by collapsing and expelling gas, offering a new means to induce gas desorption.

However, despite their appealing adsorption properties, such MOFs with pliable struts and backbones are arguably infeasible for practi- cal applications: repeated dynamics eventually lead to structural failure and degradation. ${ }^{7}$ In addition, engineering challenges are associated with designing a pressure vessel that accommodates the volume changes of flexible MOFs with lattice distortions. ${ }^{13}$

A vision towards robust, high-fidelity, fixedvolume MOFs that retain dynamic complexity and exhibit adsorption properties inaccessible to rigid materials is to graft dynamic, mechanically interlocked molecules onto rigid MOFs, treated as scaffolds. ${ }^{7,27-29}$ In contrast to MOFs with pliable struts and backbones which, subject to repeated dynamics, will rupture, mechanically interlocked molecules ${ }^{30,31}$ can undergo repeated dynamics without breakage. In addition, as some mechanically interlocked molecules are influenced by light and electric field, ${ }^{27}$ integrating mechanically interlocked components into MOF scaffolds may offer new routes to modulate gas adsorption with exotic (aside from the usual temperatureand pressure-swing) external stimuli. As an example mechanically interlocked molecule, a [2]rotaxane ${ }^{27,32-34}$ consists of a long molecule (the "axle") threaded through a macrocycle (the "wheel"); bulky groups at the two ends of the axle prevent the wheel from sliding off the axle, thereby mechanically interlocking the wheel and axle. In a [2]rotaxane molecular shuttle, the wheel is free to translate along the axle, which can be decorated with functional groups that serve as "stations" for the wheel, possibly with different affinities for it. Pioneering experimental studies have demonstrated the integration of rotaxanes into MOF scaffolds, ${ }^{35-46}$ but resultant adsorption properties have not been investigated.

Metal-organic frameworks with rotaxane molecular shuttles operating in their pores, ${ }^{36}$ RMS-MOFs (RMS = rotaxane molecular shuttle), are particularly promising for exhibiting anomalous adsorption properties, which could arise from (i) energetic effects via competition between the wheel and a gas molecule for a station on the axle and (ii) entropic effects via adsorbed gas altering the translational freedom of the wheel. In 2015, Loeb and coworkers ${ }^{36}$ synthesized an RMS-MOF coined UWDM-4. ${ }^{28}$ 
The struts of UWDM-4 serve as the axle for a [2] rotaxane, whose macrocyclic wheel was shown to rapidly shuttle between the two benzimidazole stations on its strut, unimpeded owing to the porosity of the MOF. ${ }^{36}$ Fig. 1a shows the [2] rotaxane linker used to synthesize UWDM-4. To our knowledge, UWDM-4 is the only reported RMS-MOF, and its adsorption properties have not been studied.

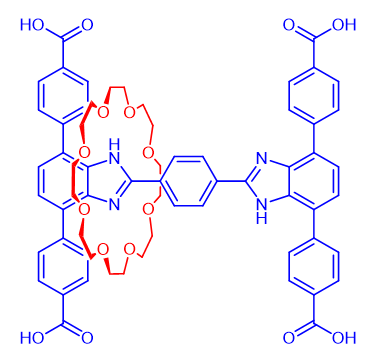

(a)

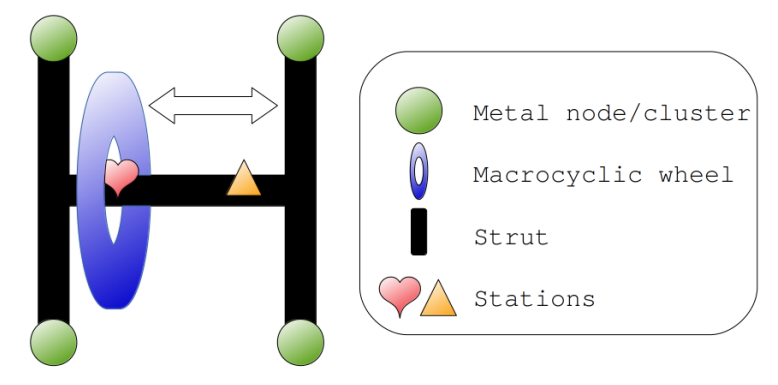

(b)

Figure 1: A MOF with a rotaxane molecular shuttle operating in its pores, an RMS-MOF. (a) The RMS-MOF linker used to synthesize UWDM-4 (blue: axle/strut; red: wheel), ${ }^{36}$ which inspired our abstraction in (b). (b) Our abstraction of the unit cell of an RMS-MOF. The wheel can translate along the rotaxane axle (= the strut of the MOF), which harbors two distinct stations $(\triangle$ and $\triangle$ ) for the wheel that also serve as adsorption sites for gas molecules. If the wheel occupies the $\nabla(\triangle)$ station, the $\triangle$ $(\odot)$ adsorption site is exposed to the gas.

Herein, we formulate and analyze a simple statistical mechanical model of an RMS-MOF and its interaction with gas molecules to investigate its adsorption properties. See Fig. 1b. Our goals are to fundamentally understand how the adsorption properties of an RMS-MOF are affected by (i) competition between the wheel and gas for stations/sites on the strut of the MOF and (ii) changes in the translational entropy of the shuttling wheel induced by the adsorption of gas. By formulating a useful abstraction of the RMS-MOF similar in spirit to the seminal Langmuir adsorption model, ${ }^{47}$ we aim to illuminate potentially anomalous or enhanced adsorption properties that could be imparted by a shuttling wheel within a MOF. Compared to a rigid, single-site Langmuir material, we find that, depending on the interactions of the wheel and gas with the stations/sites, (i) adsorption in the RMS-MOF can be more or less temperature-sensitive and (ii) more or less heat can be released upon adsorption. Interestingly, we find that the position of the wheel is a non-montonic function of temperature when the gas fiercely competes with the wheel for a station/site.

\section{Background: the Lang- muir adsorption model}

We first briefly review the classic, single-site Langmuir adsorption model, ${ }^{47}$ to which we will compare our RMS-MOF model.

The unit cell of a Langmuirian MOF offers a single, independent gas adsorption site, $\square$. See Fig. 2a. Let $n \in\{0,1\}$ be the number of adsorbed gas particles in the unit cell, our system, and $\epsilon_{\square}$ be the energy of the gas- $\square$ interaction. The energy of the system is then $E(n)=n \epsilon_{\square}$.

Immersed in a bath of gas at temperature $T$ and chemical potential $\mu$, the grand canonical partition function of the Langmuir MOF system is:

$$
\xi(\mu, T)=\sum_{n \in\{0,1\}} e^{-\beta E(n)+\beta \mu n}=1+e^{\beta\left(\mu-\epsilon_{\square}\right)},
$$

with $\beta:=1 /\left(k_{\mathrm{B}} T\right)$ and $k_{\mathrm{B}}$ the Boltzmann constant. The expected number of adsorbed gas molecules in the Langmuir MOF is:

$$
\langle n\rangle_{L}(P, T)=\sum_{n \in\{0,1\}} n \frac{e^{-\beta E(n)+\beta \mu n}}{\xi}=\frac{K_{\square} \beta P}{1+K_{\square} \beta P}
$$

We arrived at eqn. 2 by treating the gas phase as an ideal [lattice] gas, giving a relation between the pressure $P$ of the gas and its chem- 
ical potential $\mu, \beta P=e^{\beta \mu}$ (see Sec. S1). The Langmuir parameter $K_{\square} \equiv e^{-\beta \epsilon_{\square}}$ is a function of temperature and describes the affinity of the gas for the $\square$ adsorption site; the $\square$ adsorption sites are half-occupied when the density of the ideal gas phase $\beta P=1 / K_{\square}$. Fig. $2 \mathrm{~b}$ shows the shape of the Langmuir adsorption isotherm in eqn. 2, a monotonically increasing, concave function that approaches 1 (fully occupancy) as $K_{\square} \beta P \rightarrow \infty$. Finally, the internal energy of the Langmuirian unit cell is, intuitively, the product of the occupancy and the gas- $\square$ interaction:

$$
\begin{aligned}
\langle E\rangle_{L}(P, T) & =\sum_{n \in\{0,1\}} E(n) \frac{e^{-\beta E(n)+\beta \mu n}}{\xi} \\
& =\epsilon_{\square}\langle n\rangle_{L}(\mu, T) .
\end{aligned}
$$

This gives a differential energy of adsorption $\partial\langle E\rangle_{L} / \partial\langle n\rangle_{L}=\epsilon_{\square}$

\section{Abstracting gas adsorp- tion in an RMS-MOF}

We formulate a toy statistical mechanical model of (pure) gas adsorption in a MOF with a rotaxane molecular shuttle operating in its pores, i.e., in an RMS-MOF. Fig. 1b shows our modeling abstraction of the unit cell of the RMS-MOF structure, inspired by UWDM$4{ }^{36}$ The (bistable) rotaxane strut of the RMSMOF presents two distinct binding sites, $\varnothing$ and $\triangle$, that serve as: (i) stations for the shuttling, macrocyclic wheel mechanically interlocked with the strut and (ii) adsorption sites for the gas. Because the wheel sterically excludes gas, the position of the wheel determines which adsorption site is exposed to the gas. We take the RMS-MOF scaffold as rigid, imposing a constant volume.

\subsection{The space of microstates}

We begin by specifying the space of possible microstates of the RMS-MOF-gas system. We define the system as one unit cell of the RMSMOF crystal shown in Fig. 1b. We treat this as an independent system, thereby neglecting

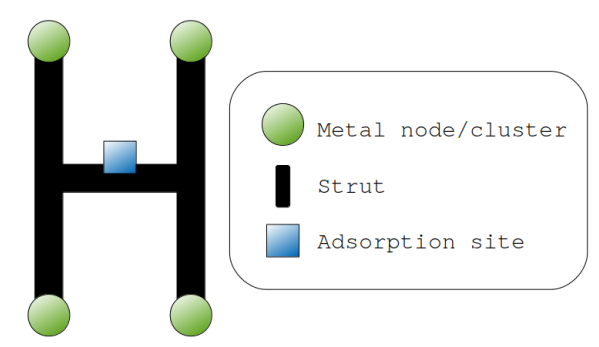

(a)

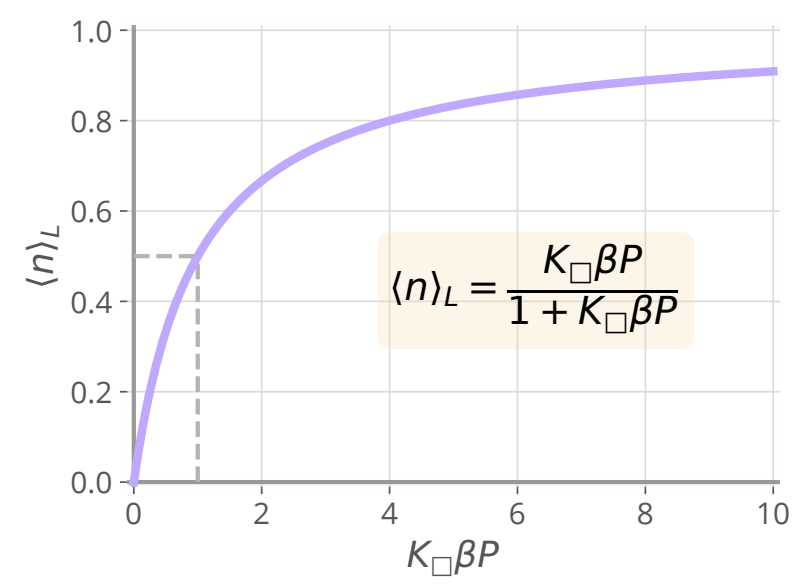

(b)

Figure 2: The single- $\square$-site Langmuir adsorption model. (a) The unit cell offers a single adsorption site, $\square$, with gas-box interaction energy $\epsilon_{\square}$. (b) The shape of the Langmuir adsorption isotherm, with $\beta P$ the density of the (ideal) bulk gas and $K_{\square} \equiv e^{-\beta \epsilon_{\square}}$ the temperature-dependent Langmuir parameter. Half-saturation of the adsorption sites occurs when $K_{\square} \beta P=1$ (dashed lines).

interactions of gas molecules and wheels with those in neighboring unit cells.

the wheel. The wheel can adopt two possible microstates: it sits at either the $\varnothing$ or $\triangle$ station. Let $w \in\{0,1\} \equiv \mathcal{W}$ denote the microstate of the wheel on the strut $(w=0 \Longrightarrow$ wheel on $\oslash, w=1 \Longrightarrow$ wheel on $\triangle)$.

the gas. The RMS-MOF unit cell can fit only one gas molecule inside. Let $n \in\{0,1\} \equiv \mathcal{N}$ be the number of gas molecules adsorbed. If $n=1$, the site on which the gas molecule is adsorbed is determined by the state of the wheel, $w$. If $w=0$, the $\triangle$ site is exposed to the gas. If $w=1$, the $\odot$ site is exposed to the gas.

the wheel-gas system. The set of four possible microstates of the RMS-MOF-gas system, 
$\mathcal{W} \times \mathcal{N}$, is enumerated in Tab. 1 .

Table 1: List of microstates, $\mathcal{W} \times \mathcal{N}$, and associated energies for the RMS-MOF-gas system. Gray sphere represents an adsorbed gas molecule.

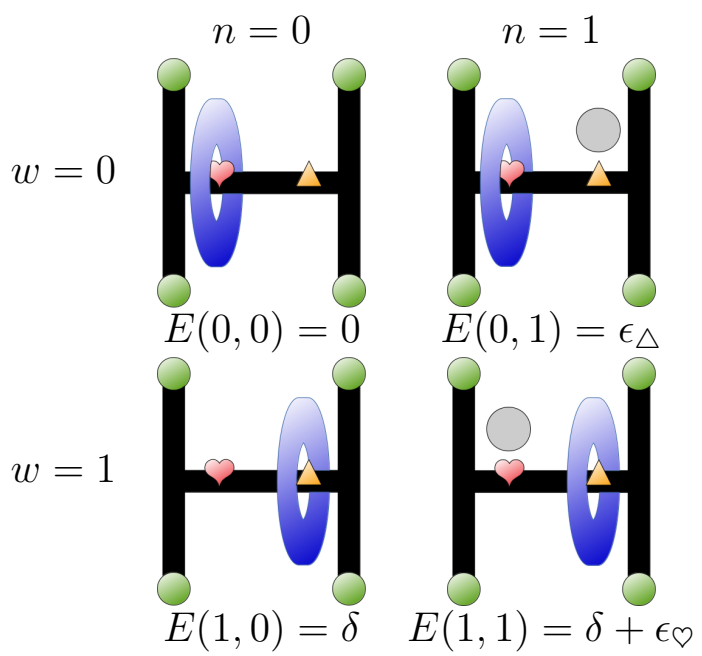

\subsection{The energy of the mi- crostates}

Next, we prescribe the energy of each microstate of the RMS-MOF-gas system. The $\varnothing$ station is the ground state of the wheel, with $\delta$ the energetic penalty for the wheel to reside at the $\triangle$ station instead. A gas molecule adsorbed on the $\varnothing$ and $\triangle$ adsorption site experiences an energy of $\epsilon_{\odot}$ and $\epsilon_{\triangle}$, respectively, owing to (van der Waals, electrostatic, etc.) interactions with the site. The energy $E$ of microstate $(w, n) \in \mathcal{W} \times \mathcal{N}$ is then:

$$
E(w, n)=w \delta+n\left[w \epsilon_{\odot}+(1-w) \epsilon_{\triangle}\right] .
$$

The first and second term arise from wheelstation and gas-station interactions, respectively. Tab. 1 illustrates the energy prescription in eqn. 5. Tab. 2 summarizes model parameters.

\subsection{RMS-MOF materials space}

Owing to the adjustable chemistry of MOFs, the energetic parameters $\delta, \epsilon_{\odot}, \epsilon_{\triangle}$ can be tuned by changing the chemistry of the RMS-MOF, particularly the chemistry of the stations $\varnothing$ and $\triangle$ and of the macrocyclic wheel. For this reason, we define RMS-MOF material space to be the set of all parameters $\left(\delta, \epsilon_{\odot}, \epsilon_{\triangle}\right)$, in the context of a particular gas. Though, we enforce (i) $\delta>0$ to avoid model redundancy that, otherwise, arises from model symmetry and (ii) $\epsilon_{\odot}, \epsilon_{\triangle}<0$ corresponding to attractive, as opposed to repulsive, site-gas interactions.

\subsection{Classification of materials space}

We classify RMS-MOFs according to the relative gas-station and wheel-station affinities, i.e., according to their location in materials space. For this classification, consider an RMS-MOFgas system with $n=1$ fixed (but $w$, the position of the wheel, can fluctuate in the $n V T$ ensemble). These classifications are depicted in Fig. 3a.

case: $\epsilon_{\odot}<\epsilon_{\triangle}$, wheel-gas competition. If the gas has a higher affinity for the $\varnothing$ site than for the $\triangle$, adsorbed gas and the wheel compete for the $\varnothing$ site. Three subcases of wheel-gas competition are ordered by increasing affinity of the gas for the $\varnothing$ station:

subcase: $\epsilon_{\triangle}<\epsilon_{\odot}+\delta$, wheel wins. The energetic preference of the gas for the $\varnothing$ site over the $\triangle$ site is insufficiently strong to offset the penalty for the wheel to occupy the $\triangle$ station. In this subcase, the ground state of the wheel is on $\varnothing$ station in both the fixed $n=0$ and fixed $n=1$ canonical ensembles.

subcase: $\epsilon_{\odot}+\delta=\epsilon_{\triangle}$, detente. The energetic preference of the gas for the $\varnothing$ site over the $\triangle$ site exactly offsets the energetic penalty for the wheel to reside on the $\triangle$ station. In this subcase, the energies of the two microstates of the fixed $n=1$ RMS-MOF-gas system are equal. Thus, in the fixed $n=1$ canonical ensemble, the wheel shares residence equally between the $\triangle$ and $\nabla$ stations.

subcase: $\epsilon_{\odot}+\delta<\epsilon_{\triangle}$, gas wins. The energetic preference of the gas for the $\nabla$ site over the $\triangle$ site is sufficiently strong to offset the penalty for the wheel to occupy the $\triangle$ station. Interestingly, in this subcase, ground state of the wheel differs between the fixed $n=0$ and fixed $n=1$ canonical ensemble. While the ground state of 
Table 2: Description of model parameters/variables

\begin{tabular}{llc} 
Type & Description & Symbol \\
\hline material parameter & energy of gas molecule adsorbed on site $\varnothing$ & $\epsilon_{\odot}$ \\
material parameter & energy of gas molecule adsorbed on site $\triangle$ & $\epsilon_{\triangle}$ \\
material parameter & energetic penalty for the wheel to occupy $\triangle$ station & $\delta$ \\
microstate variable & position of the wheel $(w=0 \Longrightarrow \varnothing, w=1 \Longrightarrow$ & $w$ \\
microstate variable & number of adsorbed gas molecules in the RMS-MOF unit cell & $n$ \\
macrostate variable & chemical potential set by bulk gas phase & $\mu$ \\
macrostate variable & thermodynamic beta $1 /\left(k_{\mathrm{B}} T\right)$ set by bulk gas phase & $\beta$
\end{tabular}

the wheel is on the $\odot$ when empty of gas, the ground state switches to the $\triangle$ when the RMSMOF is occupied by gas, so as to expose the $\varnothing$ station to the gas. See Fig. 3b.

case: $\epsilon_{\odot}=\epsilon_{\triangle}$, wheel-gas peace. The gas$\varnothing$ attraction is equal to the gas- $\triangle$ interaction.

The gas is indifferent to which adsorption site it binds; hence, there is effectively no interaction between the gas and the wheel. A symmetric $\mathrm{RMS}-\mathrm{MOF}$ that presents identical stations ( " $\triangle=\triangle$ ") exhibits wheel-gas peace.

case $\epsilon_{\triangle}<\epsilon_{\odot}$ : wheel-gas cooperation. The gas- $\triangle$ interaction is more favorable than the gas- $\odot$ interaction. Thus, the wheel and the gas energetically prefer opposite stations. We classify this as cooperation because, comparing the fixed $n=0$ and fixed $n=1$ canonical ensembles, the addition of a gas molecule increases the residence of the wheel on its preferred station, the $\varnothing$.

\section{Adsorption properties of the RMS-MOF}

Consider the model RMS-MOF unit cell immersed in and in thermodynamic equilibrium with a heat and particle bath of pure gas at temperature $T$ and chemical potential $\mu$. The chemical potential of the gas, $\mu$, is coupled to its pressure, $P$, through an equation of state, like the ideal gas law we invoke here. Our goal is to determine the gas adsorption $\langle n\rangle$, wheel residence $\langle w\rangle$, and internal energy $\langle E\rangle$ as a function of the state variables, $P$ and $T$, as well as in relation to the material parameters, $\left(\delta, \epsilon_{\odot}, \epsilon_{\triangle}\right)$.

\subsection{The grand-canonical parti- tion function}

The grand-canonical partition function of the RMS-MOF unit cell in Fig. 1b is:

$$
\begin{aligned}
\xi(\mu, T) & =\sum_{(w, n) \in \mathcal{W} \times \mathcal{N}} e^{-\beta E(w, n)+\beta \mu n} \\
& =1+e^{-\beta \delta}+e^{\beta \mu}\left(e^{-\beta \epsilon \Delta}+e^{-\beta\left(\epsilon_{\odot}+\delta\right)}\right),
\end{aligned}
$$

with $E(w, n)$ given by eqn. 5. The first two terms correspond to the two microstates with $n=0$; the latter two correspond to $n=1$.

\subsection{Gas adsorption}

The expected number of adsorbed gas particles in the RMS-MOF is:

$$
\begin{aligned}
\langle n\rangle(P, T) & =\sum_{(w, n) \in \mathcal{W} \times \mathcal{N}} n \frac{e^{-\beta E(w, n)+\beta \mu n}}{\xi} \\
& =\frac{K^{\prime} \beta P}{1+K^{\prime} \beta P},
\end{aligned}
$$

with

$$
K^{\prime}=K^{\prime}(T) \equiv \frac{1}{1+e^{-\beta \delta}} e^{-\beta \epsilon_{\triangle}}+\frac{e^{-\beta \delta}}{1+e^{-\beta \delta}} e^{-\beta \epsilon_{\odot}} .
$$

To arrive at eqn. 8 , we substituted $e^{\beta \mu}$ with $\beta P$ by treating the bulk gas phase as an ideal [lattice] gas (see Sec. S1). As each RMS-MOF unit cell in Fig. 1b is independent, $\langle n\rangle$ represents the fraction of unit cells in a macroscopic RMS-MOF crystal that are occupied by a gas molecule.

The adsorption isotherm of the RMS- 


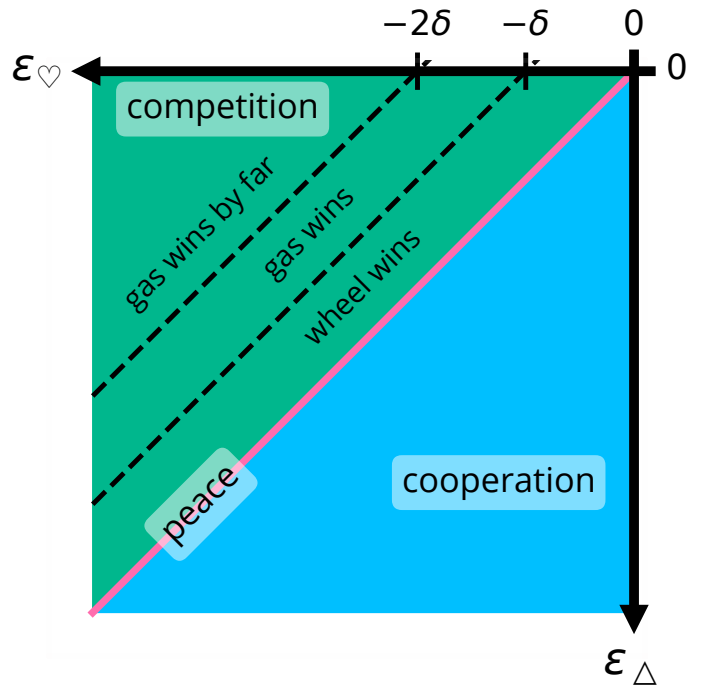

(a)

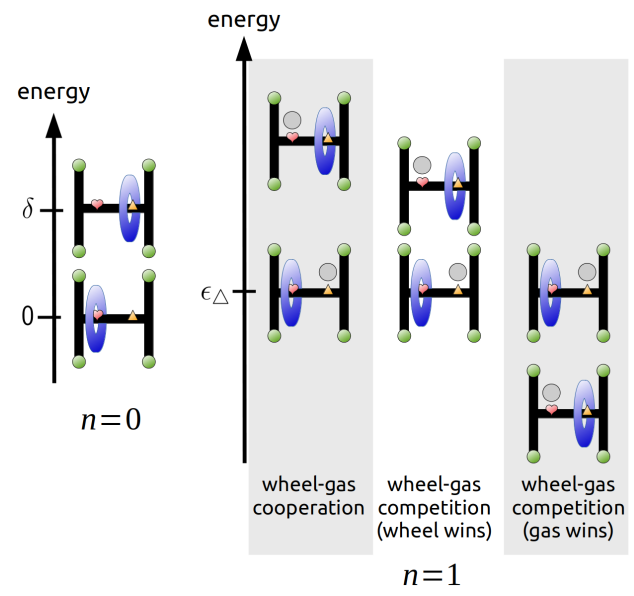

(b)

Figure 3: Classifying RMS-MOF-gas systems according to the relative gas-station and wheelstation affinities. (a) A slice of RMS-MOF materials space with fixed $\delta$, colored according to wheel-gas competition, peace, and cooperation. (b) A depiction of the relative energies of the two microstates of the RMS-MOF for fixed $n=0$ (left) and fixed $n=1$ (right). The RMS-MOF classifications are based on the fixed $n=1$ systems. Under wheel-gas competition, if the gas wins, the ground state of the wheel is on the $\triangle$ in the fixed $n=1$ canonical ensemble.

MOF, given in eqn. 8, exhibits a functional form/shape equivalent to that of a singlesite Langmuir model given in eqn. 2 and displayed in Fig. 2b. The temperature-dependent, pseudo-Langmuir parameter $K^{\prime}$ in eqn. 9 describes the effective affinity of the gas for the
RMS-MOF, with half of the RMS-MOF unit cells occupied when the density of the ideal gas phase $\beta P=1 / K^{\prime}$. Intuitively, $K^{\prime}$ is a weighted average of the Langmuir parameters of a single- $\triangle$-site and a single- $\bigcirc$-site $\mathrm{MOF}$, $K_{\triangle} \equiv e^{-\beta \epsilon_{\triangle}}$ and $K_{\odot} \equiv e^{-\beta \epsilon_{\odot}}$, respectively; the weight $\left(1+e^{-\beta \delta}\right)^{-1}$ on $K_{\triangle}$ is the probability that the wheel resides on $\varnothing$ when the RMSMOF is empty of gas, thereby exposing the $\triangle$ to the gas, while the weight on $K_{\odot}$ is the probability the wheel resides on $\triangle$ when the RMSMOF is empty of gas. However, we refer to $K^{\prime}$ as a pseudo-Langmuir parameter because its temperature-dependence is not equivalent to a single- $\square$-site Langmuir model, $K_{\square}(T)=e^{-\beta \epsilon_{\square}}$ (see Sec. 2). An implication of this, which we explore later, is that gas adsorption in the RMS-MOF scales with temperature differently than in a Langmuir material.

Note, gas adsorption in the RMS-MOF is equivalent to a Langmuir adsorption model when:

- $\delta \rightarrow \infty$, as then the wheel is fixed at the $\varnothing$ station and the $\triangle$ is invariably exposed to gas, giving a single- $\triangle$-site Langmuir model.

- $\epsilon_{\odot}=\epsilon_{\triangle}$ (wheel-gas peace), as then the gas and the wheel effectively do not interact, giving a single- $\triangle=\varnothing$-site Langmuir model.

- $\epsilon_{\triangle}=\epsilon_{\odot}+2 \delta$, as then, we will later show, the entropy of the wheel is unaffected by the adsorption of gas, giving a single-site Langmuir model with a gas-site energy of interaction of $\epsilon_{\odot}+\delta$.

Other than these cases, the temperaturedependence of gas adsorption in the RMSMOF model differs from a single-site Langmuir model, owing to the translational entropy of the wheel that is altered by the adsorption of gas. 


\subsection{The position of the wheel}

The expected value of $w$, the probability that the wheel occupies the $\triangle$ station, is:

$$
\begin{aligned}
\langle w\rangle(P, T) & =\sum_{(w, n) \in \mathcal{W} \times \mathcal{N}} w \frac{e^{-\beta E(w, n)+\beta \mu n}}{\xi} \\
& =\left(\frac{e^{-\beta \delta}}{1+e^{-\beta \delta}}\right)\left(\frac{1+e^{-\beta \Theta_{\ominus}} \beta P}{1+K^{\prime} \beta P}\right)
\end{aligned}
$$

where $K^{\prime}$ is the temperature-dependent psuedoLangmuir parameter given in eqn. 9.

We recover a two-state model for the wheel when $P=0$ and the RMS-MOF is empty of gas (fixed $n=0$ ), and when $P \rightarrow \infty$ and the RMSMOF is certainly occupied by gas (fixed $n=$ 1 ). For fixed $n=0$, the wheel toggles between the $\varnothing$ and $\triangle$ stations with energies 0 and $\delta$, respectively. For fixed $n=1$, the wheel toggles between the $\nabla$ and $\triangle$ stations, in conjunction with the adsorbed gas toggling between the $\triangle$ and $\varnothing$ sites, respectively, with energies $\epsilon_{\triangle}$ and $\epsilon_{\odot}+\delta$, respectively. This is readily apparent after using eqn. 8 to write eqn. 11 as:

$$
\begin{aligned}
\langle w\rangle= & (1-\langle n\rangle)\left(\frac{e^{-\beta \delta}}{1+e^{-\beta \delta}}\right)+ \\
& \langle n\rangle\left(\frac{e^{-\beta\left(\epsilon_{\odot}+\delta\right)}}{e^{-\beta \epsilon_{\Delta}}+e^{-\beta\left(\epsilon_{\odot}+\delta\right)}}\right) .
\end{aligned}
$$

As $P$ increases from zero and gas fills the adsorption sites in the RMS-MOF, wheel-gas competition or cooperation affects the residency of the wheels. Both eqns. 11 and 12 reveal three distinct cases illustrated in Fig. 4. If the RMS-MOF exhibits wheel-gas competition $\left(e^{-\beta \epsilon_{\odot}}>K^{\prime}\right)$, more of the wheels reside on the $\triangle$ station as gas adsorbs and "kicks" some wheels off of their energetically preferred $\varnothing$ stations. If the RMS-MOF exhibits wheel-gas peace $\left(K^{\prime}=e^{-\beta \epsilon_{\odot}}\right)$, the adsorption of gas does not influence the position of the wheels, since the gas has no energetic preference for either the $\triangle$ or $\varnothing$ adsorption sites. If the RMS-MOF exhibits wheel-gas cooperation $\left(K^{\prime}>e^{-\beta \epsilon_{\odot}}\right)$, more of the wheels reside on the $\odot$ station as gas adsorbs and restricts the wheel from exploring its higher-energy state on the $\triangle$ station while undergoing thermal fluctuations.

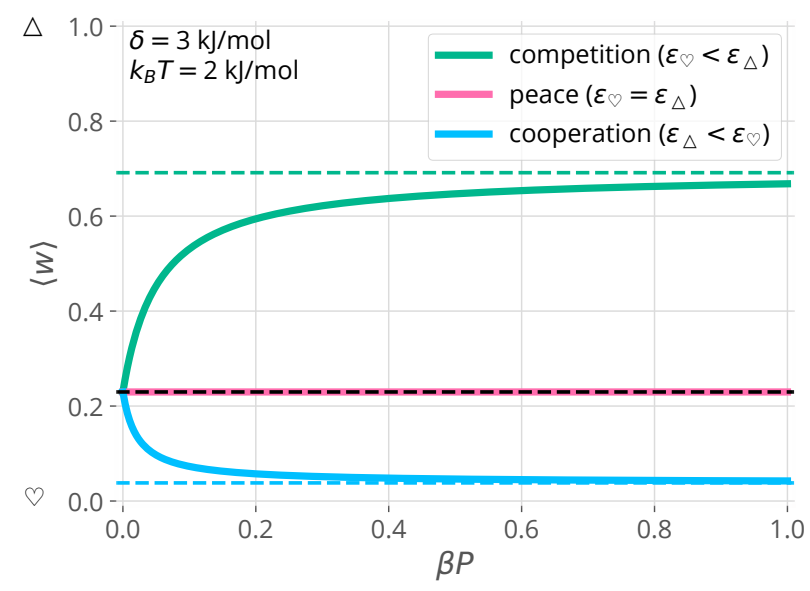

Figure 4: The fraction of the wheels in an RMS-MOF crystal that reside at their $\triangle$ station, $\langle w\rangle$, as a function of the density of the ideal gas in which the RMS-MOF is immersed, $\beta P$, at fixed temperature $(T=298 \mathrm{~K})$, for three different RMS-MOFs. Because $\delta=3$ $\mathrm{kJ} / \mathrm{mol}$ for all three RMS-MOFs, as $\beta P \rightarrow 0$, $\langle w\rangle \rightarrow e^{-\beta \delta} /\left(1+e^{-\beta \delta}\right)$ for each MOF (black, dashed line). For the RMS-MOF with wheelgas competition $\left(\epsilon_{\odot}=-10, \epsilon_{\triangle}=-5 \mathrm{~kJ} / \mathrm{mol}\right)$, more of the wheels reside at the $\triangle$ stations as gas adsorbs. For the RMS-MOF with wheelgas peace $\left(\epsilon_{\odot}=\epsilon_{\triangle}=-7 \mathrm{~kJ} / \mathrm{mol}\right)$, gas adsorption does not affect the position of the wheels. For the RMS-MOF with wheel-gas cooperation $\left(\epsilon_{\odot}=-5, \epsilon_{\triangle}=-10 \mathrm{~kJ} / \mathrm{mol}\right)$, more of the wheels reside at the $\odot$ stations as gas adsorbs. The green and blue dashed lines show $\langle w\rangle$ in the two-state model for fixed $n=1$ recovered by eqn. 12 .

The perspective of the wheel as a transition between two two-state models as gas fills the RMS-MOF in eqn. 12 reveals that the direction of the temperature-dependence of $\langle w\rangle$ can depend on the amount of gas adsorbed in the RMS-MOF. When the RMS-MOF is empty (fixed $n=0$ ), the minimum-energy state of the wheel is the $\odot$ station $(\delta>0)$. An increase in temperature induces the wheel to visit its higher energy microstate, the $\triangle$ station, more frequently. Now, consider the RMS-MOF full of gas (fixed $n=1$ ). If $\epsilon_{\Delta}<\delta+\epsilon_{\mathcal{Q}}$, the $\odot$ station is still the ground state for the wheel, and an 
increase in temperature still increases the residence of the wheel on the $\triangle$ station. However, if $\epsilon_{\curlyvee}+\delta<\epsilon_{\triangle}$ (wheel-gas competition, gas wins), the $\triangle$ station is the ground state for the wheel, so as to expose the $\nabla$ site to the gas, and, instead, an increase in temperature increases the residence of the wheel on the $\nabla$ station. Fig. 5a illustrates this case of wheel-gas competition, where the gas wins and the ground state of the wheel switches upon adding or removing a gas molecule.

Fig. 5b shows isobars of $\langle w\rangle$ for an RMSMOF with $\epsilon_{\odot}+\delta<\epsilon_{\triangle}$ (wheel-gas competition, gas wins), whose non-monotonicity can be explained by the transition of the RMSMOF from the fixed $n=1$ to the fixed $n=0$ two-state model as temperature increases from zero. For juxtaposition, the bottom, blue and top, yellow curves, respectively, show the fixed $n=0$ and fixed $n=1$ two-state models for the wheel recovered from eqn. 12. Let us follow a low-pressure isobar of $\langle w\rangle$. At low temperature, the RMS-MOF is full of gas, and an increase in temperature induces the wheel to increase residence on the $\nabla$ station, like in the fixed $n=1$ case. As the temperature increases, gas is driven from the pores of the RMS-MOF, into the gas phase. As a result, beyond some pressure, the wheel then increases its residence on the $\triangle$ station with increasing temperature, like in the fixed $n=0$ case. In the high temperature limit, $\langle w\rangle=0.5$ to maximize the entropy of the wheel. While we cannot speculate on a practical application, this non-monotonic temperature-dependence of $\langle w\rangle$, revealed by the model, is interesting and unexpected.

In Fig. fig: $\mathrm{w}_{a} n d_{n o} f_{P} T$, weshow $\langle w\rangle(P, T)$ and $\langle n\rangle(P, T)$ as heatmaps, for three different classes of RMS-MOFs.

\subsection{How gas affects the entropy of the wheel}

Under wheel-gas competition or cooperation, the adsorption of a gas molecule changes the configurational entropy of the wheel,

$S_{w}=-k_{\mathrm{B}}[\langle w\rangle \log \langle w\rangle+(1-\langle w)\rangle \log (1-\langle w\rangle)]$.
Fig. 6 illustrates how $S_{w}$ changes upon the addition of a gas molecule to the unit cell of the RMS-MOF, depending on its classification (see Fig. 3a). The curve shows $S_{w}$ at fixed $n=1$ a function of $\beta\left(\delta+\epsilon_{\odot}-\epsilon_{\triangle}\right)$, given by eqn. 13 together with eqn. 12 . Now, the x's show $S_{w}$ at fixed $n=1$ for different classes of RMSMOFs, all with $\beta \delta=3$. For comparison, the solid black circle shows $S_{w}$ at fixed $n=0$ for all of the RMS-MOFs. Given $\delta>0, S_{w}$ is less than maximal when $n=0$. Comparing $S_{w}$ for fixed $n=0$ and for fixed $n=1$, arrive at the following conclusions. Under wheel-gas cooperation, the addition of a gas molecule decreases the entropy of the wheel because it increases the residence of the wheel on the $\varnothing$. Under wheel-gas peace, the addition of a gas molecule has no effect on the entropy of the wheel. Under wheel-gas competition, the addition of a gas molecule increases the residence of the wheel on the $\triangle$ station. If the wheel wins, the entropy of the wheel increases as a result. Under the detente case, the entropy of the wheel is maximized by the addition of a gas particle. If the gas wins, the entropy could either increase or decrease; the gas decreases $S_{w}$ if it has a sufficient affinity for the $\varnothing$ site (gas wins by far) so as to severely restrict the wheel from visiting the $\varnothing$ station. The addition of a gas molecule to the unit cell of the RMS-MOF increases the configurational entropy of the wheel if and only if $-2 \delta<\epsilon_{\odot}-\epsilon_{\triangle}<0$, the gray, shaded region in Fig. 6.

\subsection{The energy change upon gas adsorption}

The expected energy of the RMS-MOF-gas system is:

$$
\begin{aligned}
\langle E\rangle(P, T) & =\sum_{(w, n) \in \mathcal{W} \times \mathcal{N}} E(w, n) \frac{e^{-\beta E(w, n)+\beta \mu n}}{\xi} \\
& =(1-\langle n\rangle)\langle E\rangle_{n=0}+\langle n\rangle\langle E\rangle_{n=1}
\end{aligned}
$$




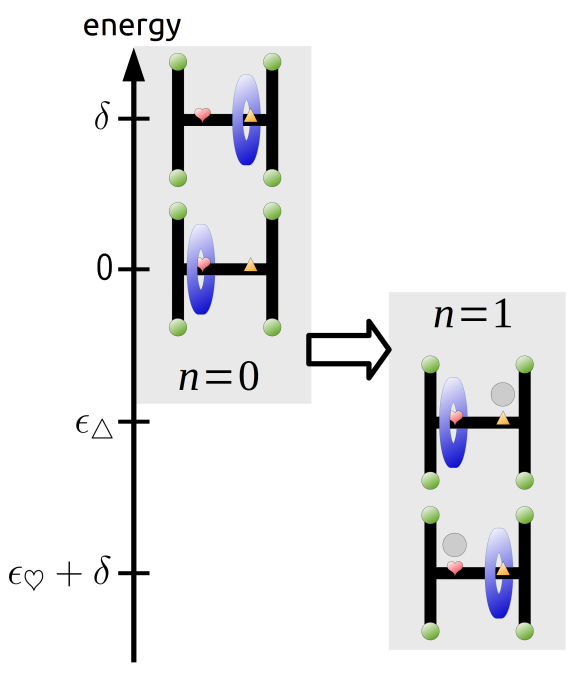

(a)

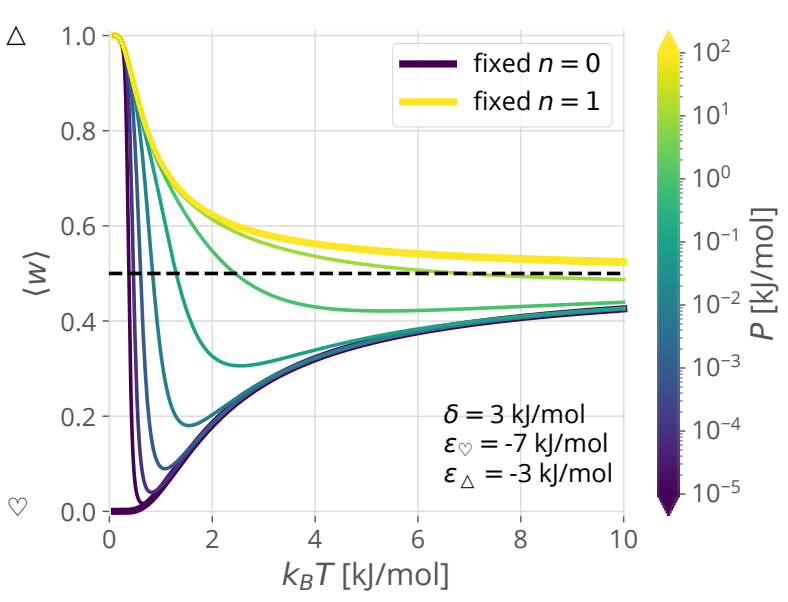

(b)

Figure 5: Under wheel-gas competition for the $\varnothing$ site, if the gas wins $\left(\epsilon_{\odot}+\delta<\epsilon_{\triangle}\right)$, the position of the wheel in the RMS-MOF responds to temperature changes differently depending on the amount of gas adsorbed. (a) In the fixed $n=0$ RMS-MOF, the $\varnothing$ station is the ground state of the wheel $(\delta>0)$. In the fixed $n=1$ RMS-MOF, the ground state of the wheel is on the $\triangle$ so as to expose the $\varnothing$ to the gas $\left(\epsilon_{\odot}+\delta<\epsilon_{\triangle}\right)$. Therefore, an increase in temperature increases wheel residence on the $\triangle$ when the RMS-MOF is empty, but instead increases wheel residence on the $\varnothing$ when the RMS-MOF is full of gas. (b) Isobars of $\langle w\rangle(T, P)$ in eqn. 11, where color indicates pressure. The two thicker curves show $\langle w\rangle$ as a function of temperature for fixed $n=0$ (bottom) and fixed $n=1$ (top), corresponding with the two two-state models in (a). The isobars of exhibit non-monotonic temperature-dependence as the RMS-MOF transitions between the two two-state models in (a) as gas fills/empties the RMS-MOF as it is cooled/warmed.

where

$$
\begin{aligned}
\langle E\rangle_{n=0}= & \delta \frac{e^{-\beta \delta}}{1+e^{-\beta \delta}} \\
\langle E\rangle_{n=1}= & \epsilon \triangle \frac{e^{-\beta \epsilon_{\triangle}}}{e^{-\beta \epsilon_{\triangle}}+e^{-\beta\left(\epsilon_{\odot}+\delta\right)}} \\
& +\left(\epsilon_{\odot}+\delta\right) \frac{e^{-\beta\left(\epsilon_{\odot}+\delta\right)}}{e^{-\beta \epsilon_{\triangle}}+e^{-\beta\left(\epsilon_{\odot}+\delta\right)}}
\end{aligned}
$$

are the expected energies of the RMS-MOF in the Canonical ensemble, with fixed $n=0$ and $n=1$, respectively. Eqn. 14 recovers the expected energy of the Langmuir model in eqn. 4 when $\delta=0$ and $\epsilon_{\odot}=\epsilon_{\triangle}=\epsilon_{\square}$.

The differential energy of adsorption is the change in energy of the [macroscopic] RMSMOF system when a molecule adsorbs from the gas phase at constant temperature. This quantity is important because it determines the heat released/consumed upon adsorption/desorption (at fixed pressure) and thus temperature-raises/drops in the adsorbent upon ad/de-sorption. ${ }^{48}$ Via eqn. 14 , the differential energy of adsorption in our RMS-MOF is

$$
\left(\frac{\partial\langle E\rangle}{\partial\langle n\rangle}\right)_{T}=\langle E\rangle_{n=1}-\langle E\rangle_{n=1}
$$

the difference between the expected energy in the fixed $n=1$ two-state model and the fixed $n=0$ two-state model. We re-write eqn. 16 to separate the contributions from the wheelstation and gas-station interactions:

$$
\begin{aligned}
\left(\frac{\partial\langle E\rangle}{\partial\langle n\rangle}\right)_{T}= & \delta\left(\frac{e^{-\beta\left(\epsilon_{\odot}+\delta\right)}}{e^{-\beta \epsilon_{\triangle}}+e^{-\beta\left(\epsilon_{\odot}+\delta\right)}}-\frac{e^{-\beta \delta}}{1+e^{-\beta \delta}}\right)+ \\
& \epsilon_{\triangle} \frac{e^{-\beta \epsilon_{\triangle}}}{e^{-\beta \epsilon_{\triangle}}+e^{-\beta\left(\epsilon_{\odot}+\delta\right)}}+ \\
& \epsilon_{\odot} \frac{e^{-\beta\left(\epsilon_{\odot}+\delta\right)}}{e^{-\beta \epsilon_{\triangle}}+e^{-\beta\left(\epsilon_{\odot}+\delta\right)}}
\end{aligned}
$$

The first term is the energy change due to the adsorbed gas altering the residency of the wheel, composed of wheel-station interactions. 


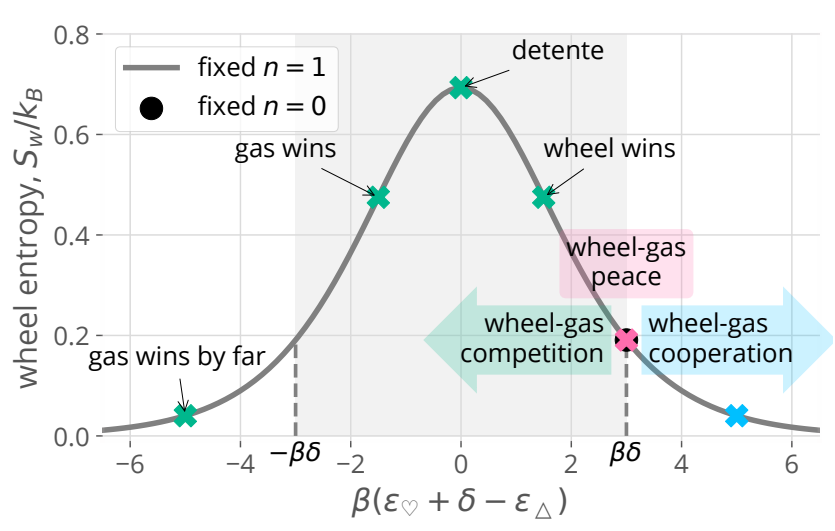

Figure 6: How gas affects the configurational entropy of the wheel, $S_{w}$ in eqn. 13. The gray curve shows $S_{w}$ at fixed $n=1$ as a function of $\beta\left(\delta+\epsilon_{\odot}-\epsilon_{\triangle}\right)$. The x's mark $S_{w}$ for fixed $n=1$ for RMS-MOFs of different classes, but all with $\beta \delta=3$. As a baseline, the black solid dot is $S_{w}$ for fixed $n=0$ for all of the RMS-MOFs. Under wheel-gas cooperation, adsorbed gas reduces the entropy of the wheel. Under wheel-gas competition, adsorbed gas can increase or decrease the entropy of the wheel, depending on how strongly the gas prefers the -exposed RMS-MOF. In the gray shaded region $-2 \delta<\epsilon_{\odot}-\epsilon_{\triangle}<0$, the addition of a gas molecule to the unit cell of the RMS-MOF increases the entropy of the wheel.

The second and third terms are the gas- $\triangle$ and gas- $\varnothing$ interactions, respectively, that appear when a gas molecule adsorbs into the RMSMOF unit cell. Eqn. 17 reveals that the energy change of the wheel, as a result of the adsorbed gas altering the residency of the wheel, contributes to the heat released/consumed upon adsorption/desorption. Under wheel-gas competition (cooperation), the wheel contributes a positive (negative) term to the differential energy of adsorption, owing to the addition of a gas particle increasing residence of the wheel on the $\triangle(\nabla)$ station.

We remark that the differential energy of adsorption in eqn. 16 is not a function of $\langle n\rangle$ because the adsorption of gas molecule $i$ in our model RMS-MOF crystal does not influence the free energy of adsorption of the next gas molecule $i+1$ that adsorbs.

\section{Comparison to a single- site Langmuir model}

We found the gas adsorption isotherm of the RMS-MOF, given in eqn. 8, to exhibit an identical functional form/shape as the single-site Langmuir adsorption isotherm derived in Sec. 2 and displayed in Fig. 2b. This raises the question: what possible advantage, for adsorptionbased engineering processes, could an RMSMOF have over an ordinary, rigid Langmuir MOF that lacks dynamic/flexible components? We next demonstrate two ways that the adsorption properties of the RMS-MOF fundamentally differ from a rigid, single-site Langmuir model that exhibits the same gas adsorption isotherm as the RMS-MOF at a reference temperature. Depending on the classification of the RMS-MOF, in the RMS-MOF:

- gas adsorption is more or less sensitive to changes in temperature. Key hint: the pseudo-Langmuir parameter $K^{\prime}$ in eqn. 9 is a more complicated function of temperature than the Langmuir parameter $K_{\square}=e^{-\beta \epsilon_{\square}}$ that appears in eqn. 2.

- more or less heat is released upon adsorption. Key hint: the wheel-station interaction contributes to the differential energy of adsorption in eqn. 17, whereas, in the Langmuir model, the differential energy of adsorption is entirely composed of the gas-site interaction.

For a suitable comparison between a given RMS-MOF and a single- $\square$-site Langmuir MOF at reference temperature $T_{0}$, we assign the gas$\square$ interaction energy $\epsilon_{\square}$ such that the Langmuir parameter $K_{\square}=e^{-\beta \epsilon_{\square}}$ in eqn. 2 is equal to pseudo-Langmuir parameter $K^{\prime}$ of the RMSMOF in eqn. 9. This equips the single- $\square$-site Langmuir material with an identical gas adsorption isotherm as the RMS-MOF at temperature $T_{0}$. We refer to such a Langmuir material as the "cognate" Langmuir material to the RMS-MOF. Figure 7 shows the $\epsilon_{\square}$ that equates the Langmuir and psuedo-Langmuir parameters, $K_{\square}\left(T_{0}\right)=K^{\prime}\left(T_{0}\right)$, and thus equates the adsorption isotherms at $T_{0}$ in the RMS-MOF and its cognate Langmuir material. 


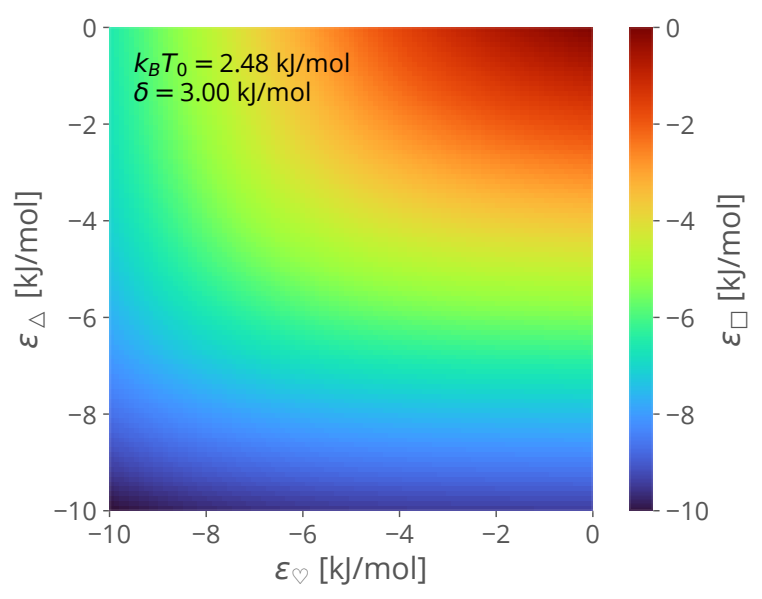

Figure 7: For a suitable comparison between a given RMS-MOF $\left(\delta, \epsilon_{\odot}, \epsilon_{\triangle}\right)$ and a single- $\square$-site Langmuir model, we assign the gas- $\square$ interaction energy, $\epsilon_{\square}$, to give the Langmuir model an adsorption isotherm identical to the adsorption isotherm of the RMS-MOF at a reference temperature $T_{0}$. The plane shows a slice of material space with $\delta$ fixed. The color indicates $\epsilon_{\square}$ such that $K_{\square}\left(T_{0}\right)=e^{-\epsilon_{\square} /\left(k_{\mathrm{B}} T_{0}\right)}=K^{\prime}\left(T_{0}\right)$.

\subsection{Temperature-sensitivity of adsorption}

Porous materials that exhibit either temperaturesensitive or temperature-insensitive adsorption are useful for engineering applications. Temperature-sensitive gas adsorption facilitates temperature-swing adsorption processes for gas storage and separations, where temperature increases are used to expel adsorbed gas from the adsorbent and regenerate it for another cycle. Temperature-insensitive gas adsorption minimizes pressure variations in adsorbed gas storage vessels exposed to a large range of temperatures. In addition, temperature-insensitive adsorption is desirable for adsorbents used in a gas sensors that operate in a range of temperatures.

The difference between the temperaturesensitivity of gas adsorption in an RMS-MOF and in its cognate single-site Langmuir material is:

$$
\begin{array}{r}
\left(\frac{\partial\langle n\rangle}{\partial T}\right)_{P}-\left(\frac{\partial\langle n\rangle_{L}}{\partial T}\right)_{P}= \\
\langle n\rangle(1-\langle n\rangle) \frac{1}{K^{\prime}}\left(\frac{d K^{\prime}}{d T}-\frac{d K_{\square}}{d T}\right),
\end{array}
$$

which has the same sign as the difference between the temperature-sensitivity of the pseudo-Langmuir parameter $K^{\prime}$ and the Langmuir parameter $K_{\square}$ displayed in Fig. 8a. Note $\frac{d K^{\prime}}{d T}, \frac{d K_{\square}}{d T} \leq 0$ for the parameters shown in Fig. 8a. In the region $-2 \delta<\epsilon_{\odot}-$ $\epsilon_{\triangle}<0$, gas adsorption in the RMS-MOF is less temperature-sensitive than in the cognate Langmuir MOF. Outside this region, gas adsorption in the RMS-MOF is more temperature-sensitive than the cognate Langmuir MOF. Note for RMS-MOFs such that $-2 \delta<\epsilon_{\odot}-\epsilon_{\triangle}<0$, the addition of a gas molecule increases the configurational entropy of the wheel (see Sec. 4.4). Therefore, if the addition of gas increases (decreases) the entropy of the wheel, then gas adsorption in the RMS-MOF is less (more) temperature-sensitive than in the cognate Langmuir material. The temperature-sensitivity of adsorption in the RMS-MOF is equal to that in its cognate Langmuir $\mathrm{MOF}$ if $\epsilon_{\triangle}=\epsilon_{\odot}$ or $\epsilon_{\triangle}=\epsilon_{\odot}+2 \delta$, since, then, the adsorption of gas does not alter the configurational entropy of the wheel.

Figs. 8b and 8c display the difference in gas adsorption between an RMS-MOF and its cognate Langmuir material in eqn. 19, for two different RMS-MOFs. At temperature $T_{0}$, by definition of the cognate Langmuir material, the RMS-MOF and the Langmuir material adsorb the same amount of gas. The RMSMOF in Fig. 8b exhibits wheel-gas competition (detente). At any given pressure, it adsorbs less gas than the cognate Langmuir MOF at temperatures lower than $T_{0}$ and more gas at temperatures higher than $T_{0}$. Therefore, as a decrease and increase in temperature, respectively, induce the materials to uptake and expel gas, respectively, gas adsorption in this RMS-MOF is less temperature-sensitive than in the cognate Langmuir material. In contrast, gas adsorption in an RMS-MOF with 
wheel-gas cooperation, as shown in Fig. 8c, is more temperature-sensitive than in the cognate Langmuir material.

\subsection{Differential energy of adsorp- tion}

Porous materials with either low or high differential energy of adsorption are useful for engineering applications. For gas storage and separations, porous materials that release minimal heat upon adsorption mitigate raises in temperature of the adsorbent bed upon gas charging, which detriment its adsorptive uptake. ${ }^{49}$ In contrast, porous materials that release a lot of heat upon adsorption are desired for thermal energy storage. ${ }^{50}$

Fig. 9 compares the differential energy of adsorption in an RMS-MOF, given by eqn. 16, with the differential energy of adsorption in its cognate Langmuir MOF, $\epsilon_{\square}$, derived in Sec. 2. Depending on the wheel-station and gas-site interactions, the differential energy of adsorption in the RMS-MOF can be more or less than in its cognate Langmuir material. If the addition of a gas particle increases the configurational entropy of the wheel (i.e., if $-2 \delta<\epsilon_{\odot}-\epsilon_{\triangle}<$ $0)$, the differential energy of adsorption in the RMS-MOF is higher (i.e., less negative) then in the Langmuir material, and less heat is released upon adsorption in the RMS-MOF. On the other hand, if the addition of a gas particle decreases the configurational entropy of the wheel, the differential energy of adsorption in the RMS-MOF is lower (i.e., more negative) than in the Langmuir material, and more heat is released upon adsorption in the RMSMOF. The differential energies of adsorption are equivalent when $\epsilon_{\odot}=\epsilon_{\triangle}$ or $\epsilon_{\triangle}=\epsilon_{\odot}+2 \delta$.

\subsection{Summary of RMS-MOF vs. Langmuir material}

Though the gas adsorption isotherm in the model RMS-MOF exhibits an identical shape as a Langmuir adsorption model (c.f. eqn. 2 and 8), the temperature-dependence of adsorption and the differential energy of adsorption differ between the RMS-MOF and its cognate Langmuir material that exhibits an identical adsorption isotherm at a reference temperature $T_{0}$. If the adsorption of a gas particle increases the configurational entropy of the wheel (i.e, if $-2 \delta<\epsilon_{\odot}-\epsilon_{\triangle}<0$ ), (i) gas adsorption is less temperature-sensitive in the RMS-MOF and (ii) less heat is released upon gas adsorption in the RMS-MOF. On the other hand, if the adsorption of a gas particle decreases the entropy of the wheel, gas adsorption is more temperature sensitive and more heat is released upon adsorption. This entropic effect could be more dramatic when fully accounting for the degrees of freedom of a wheel in a bonafide RMS-MOF, such as translation along a continuum of states on the strut, circumrotation, and motion orthogonal to the axle.

\section{Generalizing our RMS- MOF model}

To generalize our simple statistical mechanical model of an RMS-MOF, in Sec. S3 we allow multiple gas molecules to adsorb and interact in the unit cell of the RMS-MOF. We derive $\langle w\rangle$, $\langle n\rangle$, and $\langle E\rangle$ in terms of the adsorption properties of the two fixed- $w$ RMS-MOFs, where the wheel is fixed on a station. Our key findings are:

- (eqn. S17) The adsorption of gas increases the residence of the wheel on the $\varnothing(\triangle)$ station if the grand potential of the gas in the $\triangle$-exposed ( $\bigcirc$-exposed) RMS-MOF is lower than in the $\varnothing$-exposed $(\triangle$-exposed) RMSMOF.

- (eqn. S19) An increase in temperature increases residence of the wheel on the $\triangle(\nabla)$ station if the internal energy of gas in the $\triangle$-exposed RMS-MOF is lower (higher) than the internal energy of gas in the $\bigcirc$-exposed RMS-MOF plus the energetic penalty $\delta$ for the wheel to reside at the $\triangle$ station.

- (eqns. S21 and S22) Gas adsorption in the RMS-MOF is a linear interpolation of gas adsorption in the $\bigcirc$-exposed and $\triangle$-exposed RMS-MOF, weighted by $\langle w\rangle$ and $1-\langle w\rangle$, 


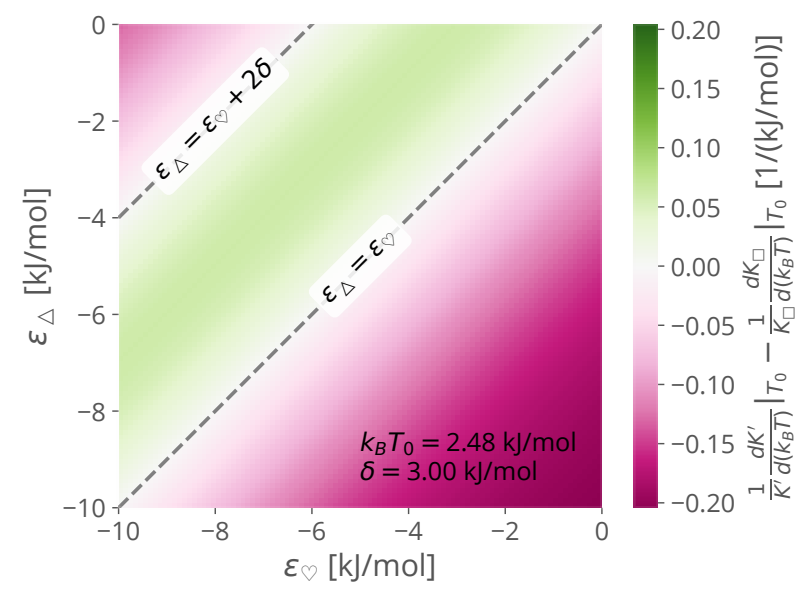

(a)

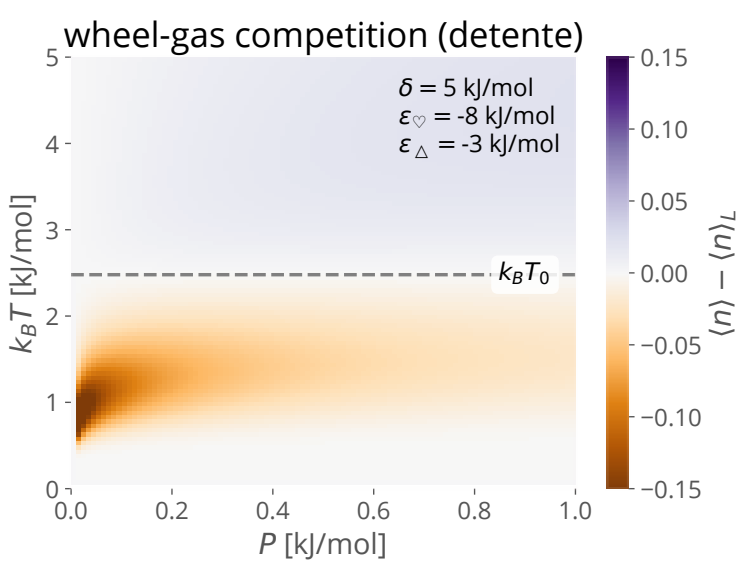

(b)

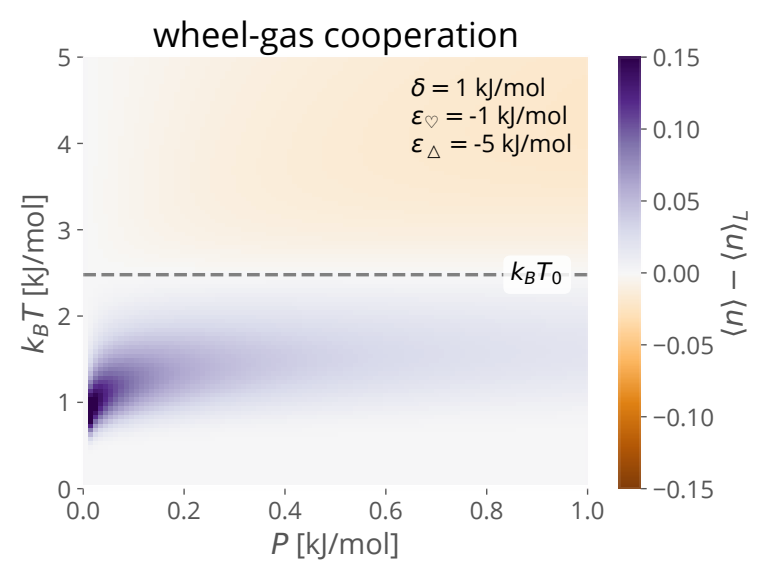

(c)

Figure 8: Comparing the temperature-sensitivity of gas adsorption in the RMS-MOF to in its cognate single-site Langmuir material, which presents an identical adsorption isotherm at reference temperature $T_{0}$. (a) The plane is a slice of RMS-MOF material space with $\delta$ fixed. The color depicts the normalized difference in the temperature-sensitivity of the psuedo-Langmuir parameter of the RMS-MOF, $K^{\prime}$, and of the Langmuir parameter of the cognate Langmuir MOF, $K_{\square}$. RMS-MOFs in the green (pink) region exhibit less (more) temperature-sensitive adsorption than their cognate Langmuir materials. (b, c) For two different RMS-MOFs, the difference in their gas adsorption and the gas adsorption in their cognate Langmuir materials, as a function of pressure and temperature.

respectively. However, the temperaturesensitivity of gas adsorption is not a linear interpolation of the temperature-sensitivity of gas adsorption in the $\nabla$ - and $\triangle$-exposed RMS-MOFs; the temperature-sensitivity of the position of the wheel also plays a role.

\section{Discussion}

Since 1918, when Langmuir introduced the single-site adsorption model, ${ }^{47}$ many molecular-level adsorption models have been developed $^{47,51}$ to explain and predict adsorption phenomena from the bottom-up. The modular and adjustable nature of MOFs has expanded the scope of complexity we can integrate into porous materials, e.g. dynamic structures that respond to gas adsorption ${ }^{8-12}$ and, consequently, exhibit unique adsorption properties. ${ }^{14}$ We may be seeing a renaissance of simple, molecular-level statistical mechanical adsorption models to explain and predict exotic 


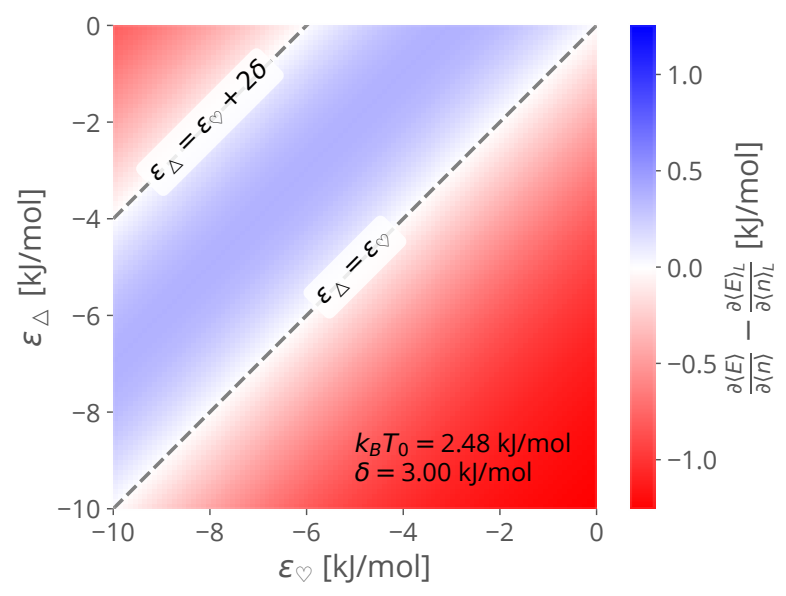

Figure 9: Comparing the differential energy of adsorption in RMS-MOFs to in their cognate single-site Langmuir materials that present an identical adsorption isotherm at reference temperature $T_{0}$. The plane is a slice of RMS-MOF materials space with $\delta$ fixed. The color depicts the difference in the differential energy of adsorption in the RMS-MOF and in the Langmuir material. RMS-MOFs in the blue (red) region release less (more) heat upon gas adsorption than their cognate Langmuir materials.

adsorption properties in these dynamic, gasresponsive materials, as exemplified by models to explain inflections in adsorption isotherms caused by gas-induced ligand rotation, ${ }^{52}$ negative gas adsorption, ${ }^{53,54}$ discontinuities in adsorption isotherms, ${ }^{55}$ gas-induced framework expansion/contraction, ${ }^{23,56-58}$ sharp steps in $\mathrm{CO}_{2}$ adsorption via the formation of ammonium carbamate chains, ${ }^{59}$ etc.

In this work, we developed a toy statistical mechanical model of gas adsorption in a metalorganic framework harboring a rotaxane molecular shuttle in its pores, an RMS-MOF. We formulated the simple abstraction of an RMSMOF in Fig. 1b similar in spirit to the seminal Langmuir adsorption model, with the aim of qualitatively exploring the adsorption properties that could be imparted to a MOF by a shuttling wheel in its pores. Our model incorporates two salient features of an RMS-MOF: (i) the translational freedom of the shuttling wheel and (ii) wheel/gas competition for the stations/sites on the strut, and provides use- ful insights into how these features determine the amount of gas adsorbed, the residency of the wheel, and the differential energy of adsorption. While we found the gas adsorption isotherm of the RMS-MOF to exhibit an identical shape as a single-site Langmuir model, the shuttling wheel in the RMS-MOF affects (i) the temperature-sensitivity of gas adsorption and (ii) the differential energy of adsorption. Compared to the cognate Langmuir material with an identical adsorption isotherm, depending on the interaction of the wheel and gas for the stations/sites, (i) gas adsorption can be more or less temperature-sensitive and (ii) more or less heat can be released upon adsorption. Both properties are useful for engineering applications and emanate from the change in the configurational entropy of the shuttling wheel caused by the adsorption of gas. Finally, we found that if the gas fiercely competes the wheel for its favorite station, temperature changes affect the position of the wheel nonmonotonically.

Regarding the pursuit of RMS-MOFs for storing and separating gases, we have an "elephant in the room": the elephant is the macrocycle wheel wrapped around the strut of the MOF scaffold, and the room is the pore space in the MOF. This large macrocycle wheel occupies precious porosity that could otherwise host another gas molecule; the wheel imposes the limitation that fewer adsorption sites for gas molecules can be packed into a fixed volume of MOF. Therefore, the necessarily reduced porosity of a RMS-MOF as well as its synthetic complexity must be compensated for by its enhanced adsorption properties to see application in gas storage and separations.

Our parsimonious toy model neglects many features of an RMS-MOF that could affect its adsorption properties, such as the continuum of wheel states along the strut, multiple gas molecules adsorbing in the unit cell and interacting with each other, adsorption sites on the MOF other than the two primary stations, interactions of gas molecules and wheels with those in neighboring unit cells, etc. Our simple model is a starting point for charting the properties that could be imparted into a MOF 
by a rotaxane molecular shuttle in its pores. It is a good approximation when the strut offers two distinct stations/sites that strongly attract the wheel/gas and thus dominate the thermodynamics.

Future work remains to chart further exotic adsorption properties offered by metal-organic frameworks harboring rotaxane molecular shuttles. To confirm our predictions herein, heroic experimental efforts are needed to synthesize a porous, activated RMS-MOF, measure its gas adsorption properties, and characterize in situ the internal state of its macrocycle wheel as a function of gas adsorption. Thus far, only one MOF harboring a rotaxane shuttle has been synthesized. ${ }^{36}$ Some remaining knowledge gaps could be filled by extending our model. First, our toy model could be decorated with complexity to account for other molecular features that can be engineered into RMS-MOFs: (i) a radially asymmetric macrocycle on the rotaxane, whose rotational conformations expose different functional groups to the stations (see Ref. 44), (ii) multiple stations [and, thus, adsorption sites] on the axle of the rotaxane, (iii) multiple macrocycle wheels threaded around the axle of the rotaxane, ${ }^{60}$ and (iv) arranging molecular shuttles in a MOF in a more complicated topology to allow coupling between neighboring molecular shuttles (inspired by Ref. 61) and (v) allowing multiple gas molecules to adsorb in a unit cell. Outside the context of MOFs, Sevick and Williams developed and analyzed a statistical mechanical model of radially asymmetric wheels $^{62}$ [case (i)] and multiple wheels ${ }^{63-65}$ [case (iii)] in a rotaxane molecular shuttle and uncovered interesting behavior. Second, analyzing our model RMS-MOF immersed in a mixture of gases could reveal enhanced selectivity arising from the molecular shuttle. Intriguingly, gas adsorption in the RMS-MOF in Fig. 1b bears some resemblance to biomolecular recognition where e.g. a ligand binds to a protein and induces a shift in its conformational ensemble. ${ }^{66}$ Third, modeling can shed light on the interplay of external stimuli and adsorption in MOFs with stimuli-responsive rotaxanes ${ }^{67}$ integrated into them. Fourth, atomistic models of RMS-MOFs could explore the practical con- straints on material space $\left(\delta, \epsilon_{\odot}, \epsilon_{\triangle}\right)$ and suggest explicit RMS-MOF chemistry to pursue for anomalous adsorption properties.

Acknowledgement C.M.S acknowledges the National Science Foundation for support under Grant No. 1920945. Thanks to Adrian Henle for the drawing in Fig. 1a.

\section{References}

(1) Morris, R. E.; Wheatley, P. S. Gas storage in nanoporous materials. Angewandte Chemie International Edition 2008, 4\%, 4966-4981.

(2) Li, J.-R.; Kuppler, R. J.; Zhou, H.-C. Selective gas adsorption and separation in metal-organic frameworks. Chemical Society Reviews 2009, 38, 1477-1504.

(3) Kreno, L. E.; Leong, K.; Farha, O. K. et al. Metal-organic framework materials as chemical sensors. Chemical Reviews 2011, 112, 1105-1125.

(4) Yaghi, O. M.; O’Keeffe, M.; Ockwig, N. W. et al. Reticular synthesis and the design of new materials. Nature 2003, 423, 705-714.

(5) Furukawa, H.; Cordova, K. E.; OKeeffe, M. et al. The chemistry and applications of metal-organic frameworks. Science 2013, 341, 1230444.

(6) Moghadam, P. Z.; Li, A.; Wiggin, S. B. et al. Development of a Cambridge Structural Database Subset: A Collection of Metal-Organic Frameworks for Past, Present, and Future. Chemistry of Materials 2017, 29, 2618-2625.

(7) Deng, H.; Olson, M. A.; Stoddart, J. F. et al. Robust dynamics. Nature Chemistry 2010, 2, 439-443.

(8) Horike, S.; Shimomura, S.; Kitagawa, S. Soft porous crystals. Nature Chemistry 2009, 1, 695-704. 
(9) Schneemann, A.; Bon, V.; Schwedler, I. et al. Flexible metal-organic frameworks. Chemical Society Reviews 2014, 43, 60626096.

(10) Coudert, F.-X. Responsive metal-organic frameworks and framework materials: under pressure, taking the heat, in the spotlight, with friends. Chemistry of Materials 2015, 27, 1905-1916.

(11) Chang, Z.; Yang, D.-H.; Xu, J. et al. Flexible metal-organic frameworks: recent advances and potential applications. Advanced Materials 2015, 27, 5432-5441.

(12) Lee, J. H.; Jeoung, S.; Chung, Y. G. et al. Elucidation of flexible metal-organic frameworks: Research progresses and recent developments. Coordination Chemistry Reviews 2019, 389, 161-188.

(13) Krause, S.; Hosono, N.; Kitagawa, S. Chemistry of Soft Porous CrystalsStructural Dynamics and Gas Adsorption Properties. Angewandte Chemie 2020,

(14) Coudert, F.-X.; Evans, J. D. Nanoscale metamaterials: Meta-MOFs and framework materials with anomalous behavior. Coordination Chemistry Reviews 2019, 388, 48-62.

(15) Winston, E. B.; Lowell, P. J.; Vacek, J. et al. Dipolar molecular rotors in the metal-organic framework crystal IRMOF2. Physical Chemistry Chemical Physics 2008, 10, 5188-5191.

(16) Krause, S.; Evans, J. D.; Bon, V. et al. Towards general network architecture design criteria for negative gas adsorption transitions in ultraporous frameworks. Nature Communications 2019, 10, 1-12.

(17) Serre, C.; Millange, F.; Thouvenot, C. et al. Very Large Breathing Effect in the First Nanoporous Chromium (III)-Based Solids: MIL-53 or $\mathrm{Cr}^{\mathrm{III}}$
$(\mathrm{OH}) \cdot\left\{\mathrm{O}_{2} \mathrm{C}-\mathrm{C}_{6} \mathrm{H}_{4}-\mathrm{CO}_{2}\right\} \cdot\left\{\mathrm{HO}_{2} \mathrm{C}_{-} \mathrm{C}_{6} \mathrm{H}_{4}{ }^{-}\right.$ $\left.\mathrm{CO}_{2} \mathrm{H}\right\}_{x} \cdot \mathrm{H}_{2} \mathrm{O}_{y}$. Journal of the American Chemical Society 2002, 124, 13519 13526.

(18) Kitaura, R.; Seki, K.; Akiyama, G. et al. Porous Coordination-Polymer Crystals with Gated Channels Specific for Supercritical Gases. Angewandte Chemie International Edition 2003, 42, 428-431.

(19) Elsaidi, S. K.; Mohamed, M. H.; Simon, C. M. et al. Effect of ring rotation upon gas adsorption in SIFSIX-3-M $(\mathrm{M}=\mathrm{Fe}, \mathrm{Ni})$ pillared square grid networks. Chemical Science 2017, 8, 2373-2380.

(20) Mason, J. A.; Oktawiec, J.; Taylor, M. K. et al. Methane storage in flexible metalorganic frameworks with intrinsic thermal management. Nature 2015, 527, 357-361.

(21) Krause, S.; Bon, V.; Senkovska, I. et al. A pressure-amplifying framework material with negative gas adsorption transitions. Nature 2016, 532, 348.

(22) Boutin, A.; Springuel-Huet, M.-A.; Nossov, A. et al. Breathing Transitions in MIL-53 (Al) Metal-Organic Framework Upon Xenon Adsorption. Angewandte Chemie International Edition 2009, 48, 8314-8317.

(23) Ghysels, A.; Vanduyfhuys, L.; Vandichel, M. et al. On the thermodynamics of framework breathing: A free energy model for gas adsorption in MIL53. The Journal of Physical Chemistry $C$ 2013, 117, 11540-11554.

(24) Evans, J. D.; Bocquet, L.; Coudert, F.-X. Origins of negative gas adsorption. Chem 2016, 1, 873-886.

(25) Choi, H. J.; Dinca, M.; Long, J. R. Broadly hysteretic $\mathrm{H}_{2}$ adsorption in the microporous metal- organic framework $\mathrm{Co}$ (1, 4-benzenedipyrazolate). Journal of the American Chemical Society 2008, 130, 7848-7850. 
(26) Chang, K.; Talu, O. Behavior and performance of adsorptive natural gas storage cylinders during discharge. Applied Thermal Engineering 1996, 16, 359 - 374.

(27) Coskun, A.; Banaszak, M.; Astumian, R. D. et al. Great expectations: can artificial molecular machines deliver on their promise? Chemical Society Reviews 2012, 41, 19-30.

(28) P. Martinez-Bulit, S. J. L., A. J. Stirk Rotors, Motors, and Machines Inside MetalOrganic Frameworks. Trends in Chemistry 2019,

(29) Krause, S.; Feringa, B. L. Towards artificial molecular factories from frameworkembedded molecular machines. Nature Reviews Chemistry 2020, 1-13.

(30) Stoddart, J. F. The chemistry of the mechanical bond. Chemical Society Reviews 2009, 38, 1802-1820.

(31) Bruns, C. J.; Stoddart, J. F. The Nature of the Mechanical Bond: From Molecules to Machines; John Wiley \& Sons, 2016.

(32) Anelli, P. L.; Spencer, N.; Stoddart, J. F. A molecular shuttle. Journal of the American Chemical Society 1991, 113, 51315133 .

(33) Balzani, V.; Credi, A.; Raymo, F. M. et al. Artificial molecular machines. Angewandte Chemie International Edition 2000, 39, 3348-3391.

(34) Abendroth, J. M.; Bushuyev, O. S.; Weiss, P. S. et al. Controlling motion at the nanoscale: rise of the molecular machines. ACS Nano 2015, 9, 7746-7768.

(35) Loeb, S. J. Metal-organic rotaxane frameworks; MORFs. Chemical Communications 2005, 1511-1518.

(36) Zhu, K.; O'Keefe, C. A.; Vukotic, V. N. et al. A molecular shuttle that operates inside a metal-organic framework. Nature Chemistry 2015, 7, 514-519.
(37) Coskun, A.; Hmadeh, M.; Barin, G. et al. Metal-Organic Frameworks Incorporating Copper-Complexed Rotaxanes. Angewandte Chemie International Edition 2012, 51, 2160-2163.

(38) Farahani, N.; Zhu, K.; O’Keefe, C. A. et al. Thermally Driven Dynamics of a Rotaxane Wheel about an Imidazolium Axle inside a Metal-Organic Framework. ChemPlusChem 2016, 81, 836-841.

(39) Vukotic, V. N.; Loeb, S. J. Coordination polymers containing rotaxane linkers. Chemical Society Reviews 2012, 41, 58965906.

(40) Zhu, K.; Vukotic, V. N.; OKeefe, C. A. et al. Metal-organic frameworks with mechanically interlocked pillars: controlling ring dynamics in the solid-state via a reversible phase change. Journal of the American Chemical Society 2014, 136, 7403-7409.

(41) Vukotic, V. N.; OKeefe, C. A.; Zhu, K. et al. Mechanically interlocked linkers inside metal-organic frameworks: effect of ring size on rotational dynamics. Journal of the American Chemical Society 2015, 137, 9643-9651.

(42) Loeb, S. J. Rotaxanes as ligands: from molecules to materials. Chemical Society Reviews 2007, 36, 226-235.

(43) Knight, L. K.; Vukotic, V. N.; Viljoen, E. et al. Eliminating the need for independent counterions in the construction of metal-organic rotaxane frameworks (MORFs). Chemical Communications 2009, 5585-5587.

(44) Baggi, G.; Loeb, S. J. Rotationally Active Ligands: Dialing-Up the Coconformations of a [2] Rotaxane for Metal Ion Binding. Angewandte Chemie International Edition 2016, 55, 12533-12537.

(45) Yang, Y.-D.; Fan, C.-C.; Rambo, B. M. et al. Multicomponent Self-Assembled Metal-Organic [3] Rotaxanes. Journal of 
the American Chemical Society 2015, 137, 12966-12976.

(46) Saura-Sanmartin, A.; MartinezCuezva, A.; Bautista, D. et al. CopperLinked Rotaxanes for the Building of Photoresponsive Metal Organic Frameworks with Controlled Cargo Delivery. Journal of the American Chemical Society $\mathbf{0}, 0$, null.

(47) Swenson, H.; Stadie, N. P. Langmuir's theory of adsorption: A centennial review. Langmuir 2019, 35, 5409-5426.

(48) Torres-Knoop, A.; Poursaeidesfahani, A.; Vlugt, T. J. et al. Behavior of the enthalpy of adsorption in nanoporous materials close to saturation conditions. Journal of Chemical Theory and Computation 2017, 13, 3326-3339.

(49) Chang, K.; Talu, O. Behavior and performance of adsorptive natural gas storage cylinders during discharge. Applied Thermal Engineering 1996, 16, 359-374.

(50) Kohler, T.; Müller, K. Influence of different adsorbates on the efficiency of thermochemical energy storage. Energy Science $\mathbb{E}$ Engineering 2017, 5, 21-29.

(51) Hill, T. L. An introduction to statistical thermodynamics; Courier Corporation, 1986.

(52) Simon, C. M.; Braun, E.; Carraro, C. et al. Statistical mechanical model of gas adsorption in porous crystals with dynamic moieties. Proceedings of the $\mathrm{Na}$ tional Academy of Sciences 2017, 114, E287-E296.

(53) Manos, G.; Dunne, L. Predicting the features of methane adsorption in large pore metal-organic frameworks for energy storage. Nanomaterials 2018, 8, 818 .

(54) Evans, J. D.; Krause, S.; Kaskel, S. et al. Exploring the thermodynamic criteria for responsive adsorption processes. Chemical Science 2019, 10, 5011-5017.
(55) Dunne, L. J.; Manos, G. Exact matrix treatment of an osmotic ensemble model of adsorption and pressure induced structural transitions in metal organic frameworks. Dalton Transactions 2016, 45, 4213-4217.

(56) Dunne, L. J.; Manos, G. Statistical mechanics of binary mixture adsorption in metal-organic frameworks in the osmotic ensemble. Philosophical Transactions of the Royal Society A: Mathematical, Physical and Engineering Sciences 2018, 376, 20170151.

(57) Triguero, C.; Coudert, F.-X.; Boutin, A. et al. Mechanism of breathing transitions in metal-organic frameworks. The Journal of Physical Chemistry Letters 2011, 2, 2033-2037.

(58) Witman, M.; Ling, S.; Stavila, V. et al. Design Principles for the Ultimate Gas Deliverable Capacity Material: Nonporous to Porous Deformations Without Volume Change. 2020,

(59) Kundu, J.; Stilck, J. F.; Lee, J.-H. et al. Cooperative gas adsorption without a phase transition in metal-organic frameworks. Physical Review Letters 2018, 121, 015701.

(60) Fang, L.; Olson, M. A.; Benítez, D. et al. Mechanically bonded macromolecules. Chemical Society Reviews 2010, 39, 17-29.

(61) Reddy, P.; Sevick, E. M.; Williams, D. R. Triangular cyclic rotaxanes: Size, fluctuations, and switching properties. Proceedings of the National Academy of Sciences 2018, 115, 9367-9372.

(62) Sevick, E. M.; Williams, D. R. Conformational isomers of linear rotaxanes. The Journal of Chemical Physics 2014, 141, 114904.

(63) Sevick, E. M.; Williams, D. Pistonrotaxanes as molecular shock absorbers. Langmuir 2010, 26, 5864-5868. 
(64) Sevick, E. M.; Williams, D. R. A pistonrotaxane with two potential stripes: Force transitions and yield stresses. Molecules 2013, 18, 13398-13409.

(65) Sevick, E. M.; Williams, D. R. A TwoStroke, Two-Cylinder Piston Rotaxane Motor. ChemPhysChem 2016, 17, 19271933.

(66) Boehr, D. D.; Nussinov, R.; Wright, P. E. The role of dynamic conformational ensembles in biomolecular recognition. $\mathrm{Na}$ ture Chemical Biology 2009, 5, 789.

(67) Xue, M.; Yang, Y.; Chi, X. et al. Development of pseudorotaxanes and rotaxanes: from synthesis to stimuli-responsive motions to applications. Chemical Reviews 2015, 115, 7398-7501. 